\title{
Testing the feasibility and effects of a self-management support intervention for patients with cancer and their family caregivers to reduce pain and related symptoms (ANtiPain): Study protocol of a pilot study
}

\author{
Antje Koller ${ }^{1,2,3}$, Monika Hasemann ${ }^{1,2}$, Karin Jaroslawski ${ }^{2}$, Sabina De Geest ${ }^{3}$, Gerhild Becker ${ }^{2}$ \\ ${ }^{1}$ Department of Internal Medicine, Medical Center-University of Freiburg, Freiburg, Germany \\ ${ }^{2}$ Department of Palliative Care, Medical Center-University of Freiburg, Freiburg, Germany \\ ${ }^{3}$ Institute of Nursing Science, Faculty of Medicine, University of Basel, Basel, Switzerland \\ Email: antje.koller@uniklinik-freiburg.de \\ Received 21 November 2013; revised 3 January 2014; accepted 23 January 2014 \\ Copyright (C) 2014 Antje Koller et al. This is an open access article distributed under the Creative Commons Attribution License, \\ which permits unrestricted use, distribution, and reproduction in any medium, provided the original work is properly cited. In accor- \\ dance of the Creative Commons Attribution License all Copyrights (C) 2014 are reserved for SCIRP and the owner of the intellectual \\ property Antje Koller et al. All Copyright (C) 2014 are guarded by law and by SCIRP as a guardian.
}

\section{ABSTRACT}

Despite effective treatment options, more than $40 \%$ of cancer patients receive inadequate pain management. Our previous pilot study resulted in substantial adaptations of a cancer pain self-management intervention, the German PRO-Self ${ }^{\odot}$ Plus Pain Control Program originally developed in the United States. This program will be implemented into clinical practice at the Medical Center-University of Freiburg. The purpose of this multiple methods pilot study is to test the implementation regarding feasibility and effects in clinical practice. In a randomized, wait-list controlled pilot study, adult oncology in-patients of a palliative care consultation service with pain $>3 / 10$ will be recruited. The intervention will be performed by a specialized advanced practice nurse with an inhospital visit and, after discharge, via phone calls and visits. The follow-up will be personalized according to a clinical algorithm that factors in pain intensity, satisfaction with pain management, and patient adherence. The intervention includes structured and tailored components and is based on three key strategies: information, skill building and nurse coaching. The specific aims of this study are threefold: 1) to test the feasibility of the study and intervention procedures; 2) to establish effect sizes of main outcome variables (e.g. decrease pain intensity, reduce the number of patients with pain as main symptom) for subsequent power calculation; 3) to explore participants' experiences with pain self-management support and their view of burden and benefit from study participation in a qualitative substudy. During the study period, which includes three data collection time points (T0 before, T1 one week and T2 six weeks after discharge), data will be collected via field notes of study nurses and questionnaires of patients. The results of this pilot study will build the basis for a larger comparative effectiveness study in which long term outcomes of a cancer pain self-management intervention in clinical practice will be evaluated.

\section{KEYWORDS}

Neoplasms; Pain; Patient Education; Pilot

Randomized Controlled Trial

\section{INTRODUCTION}

Between $30 \%$ and $75 \%$ of cancer patients experience pain, depending on the type and stage of the illness $[1,2]$. Although effective treatment options exist, more than $40 \%$ of patients with cancer-related pain do not receive adequate pain management [3]. Factors that impede pain treatment are commonly referred to as barriers to pain management [4]. Because oncology treatment takes place more and more in the outpatient setting, pain is managed mainly at home. As a result, patients' self-management plays a central role in cancer pain treatment and cancer pain self-management support interventions are gaining increasing attention [5-8]. While statistically significant decreases in pain intensity are seen across studies, the effects in terms of being clinically meaningful remain in the moderate range. Until now, interventions have varied 
greatly in design, duration, and content, and it remains unclear which types of interventions are most effective. In addition, clinical feasibility has not yet been the focus of research for pain self-management interventions [5-8].

\subsection{Development of the Self-Management Intervention}

Interventions to support cancer pain self-management are largely unknown in German-speaking populations. Therefore, for a previous pilot study, we translated and adapted the PRO-SELF ${ }^{\odot}$ Plus Pain Control Program (PCP) by Miaskowski et al. [9]. This program was found to be effective in a large RCT in the United States, and additional analyses indicated that the revised version, the PROSELFC Plus PCP, may show even higher effects $[10,11]$. Furthermore, the authors agreed to collaborate closely.

In a pilot RCT at the Tumorzentrum Ludwig Heilmeyer - Comprehensive Cancer Center Freiburg (CCCF, $\mathrm{N}=39$ ), we compared the German PRO-SELF ${ }^{\odot}$ Plus PCP with standard care. The German PRO-SELF ${ }^{\complement}$ Plus PCP was a 10-week program based on three key strategies (information, skill building, and nurse coaching) with structured and tailored components. We tested the feasibility of instruments and study procedures and calculated effect sizes for pain intensity [12,13]. Effect size calculations showed that on the 0 - 10 numeric rating scale (NRS) the mean difference of change after 10 weeks was moderate for average pain $(-0.55$ [95\% CI: $-2.31 / 1.20])$. For worst pain, the effect size was -0.73 [95\% CI: $-2.74 / 1.28]$, and the group-by-time effect was not statistically significant $[12,13]$. Using qualitative methods, we explored patients' and family caregivers' experiences with pain management and the intervention along with their view of burden and benefit from study participation [14,15]. Qualitative findings showed that the focus on cancer pain self-management was novel and welcomed by participants [14]. However, patients continued to be ambivalent about their analgesic use, balancing the need for adequate pain relief with the wish to avoid analgesics. Individually tailored counseling helped these patients to adapt to the situation [15].

\subsection{Implications of the First Pilot Study for the Proposed Project}

The pilot study provided us with the opportunity to learn from methodological difficulties [13]. Although the transfer and pilot testing of the German PRO-Self ${ }^{\odot}$ Plus PCP was quite successful and the feasibility of research and intervention procedures was established, a number of aspects warrant critical consideration. A low response rate indicates that the invitation to participate needs to be more closely linked to clinical practice. A high attrition rate (18\% in six weeks; $35 \%$ in 22 weeks) may be in- evitable and should be considered for sample size calculations.

Furthermore, it appears that the dynamic nature of cancer pain was not completely accounted for within the fixed structure of the intervention. We thus decided to break up the structure of the PRO-SELF ${ }^{\odot}$ Plus PCP and tailor it more closely to patients' individual pain situations by developing a precise clinical algorithm for follow-up. In addition, this new structure facilitated the clinical feasibility of the intervention.

Some side effects of cancer treatment (e.g., nausea) will be addressed because they severely impacted pain self-management. Most patients in the pilot study took rather low doses of opioid analgesics for their cancer pain, and daily opioid intake did not increase over time. The intervention will therefore be combined with a palliative care consultation in which state-of-the-art medical treatment will be prescribed. Furthermore, patients' adherence to prescriptions and the intervention should be evaluated more regularly in the nurse coaching component.

Regarding outcome assessments, results showed that reduction of pain intensity as a primary outcome may not have comprehensively reflected the effect of the pain self-management intervention [13]. Patients experienced more than one symptom in our previous study. Subsequently, health-related quality of life was not affected by the intervention [12]. Likewise, it was difficult to assess the influence of pain on patients' functioning. In addition to pain, several other symptoms (e.g. fatigue) often interfered with functioning, and patients had difficulties distinguishing between the symptoms' effects on functioning [13]. In contrast, the self-developed item "The pain hindered me to do the things that I wanted to do today" showed a significant group-by-time interaction effect between the intervention and the control group at week 10 ( $p=0.02$; Cohen's $d=-0.54$ ) [12]. The proposed study may be used to evaluate suitable primary and secondary outcomes. However, patient burden was an issue mentioned by patients in our last study. Therefore, patients should be provided a comprehensive choice of influencing secondary outcomes without having to complete additional questionnaires.

A first version of the advanced pain self-management intervention is now in the process of implementation in clinical practice (timetable, see Table 1). However, further pilot testing is needed to tailor the program optimally to the health care needs of the German context, not only because significant adjustments were made to the program but also because the clinical context may demand further refinement.

\subsection{Aims of the Study}

The purpose of this multiple methods pilot study is to 
Table 1. Timetable.

\begin{tabular}{|c|c|}
\hline Time & Activity or Milestone \\
\hline April 2013-Nov 2013 & $\begin{array}{l}\text { Pre-test of intervention and recruitment } \\
\text { procedures, implementation of intervention } \\
\text { (not included in project time) }\end{array}$ \\
\hline \multicolumn{2}{|c|}{ Project activities will start December 2013} \\
\hline Dec 2013-Nov 2014 & $\begin{array}{l}\text { Study coordination recruitment, data entry } \\
\text { and quality assurance, preparation of data } \\
\text { analysis (study coordinator) } \\
\text { Data collection, qualitative interviews, } \\
\text { data entry ( } 1^{\text {st }} \text { [blinded] study nurse) } \\
\text { Intervention nurse for recruitment and } \\
\text { intervention, quality assurance of protocol } \\
\text { fidelity ( } 2^{\text {nd }} \text { study nurse) }\end{array}$ \\
\hline Jan 2014-Mar 2015 & $\begin{array}{l}\text { Data entry, data quality assurance, and } \\
\text { qualitative interviews (study nurse) } \\
\text { Data analysis, preparation of publications } \\
\text { (AK) }\end{array}$ \\
\hline Apr 2015-May 2015 & Publications (AK) \\
\hline
\end{tabular}

test the implementation of an advanced cancer pain self-management support intervention in light of its feasibility and effects within the clinical context.

The primary aim is to establish key features for a randomized clinical trial of the advanced pain self-management support intervention in a clinical context. The specific aims are threefold: 1 ) to test the feasibility of the study and intervention procedures; 2) to establish effect sizes of main outcome variables (e.g. reduce pain intensity, reduce the number of patients with pain as the main symptom, increase patient satisfaction with pain management) for subsequent power calculation; 3) to explore participants' experiences with pain self-management support and their view of burden and benefit from study participation in a qualitative substudy.

\section{METHODS}

\subsection{Design}

The advanced pain self-management support intervention will be compared with standard care. A randomized, wait-list controlled pilot study will be performed with patients of the palliative care consultation service at the Medical Center-University of Freiburg (timetable see Table 1). The palliative care consultation service has been implemented to improve care for hospitalized, incurably ill patients with increased supportive care needs. This service is used by clinicians in all departments of the Medical Center. Patients will be randomized in an intervention and a wait-list control group. A qualitative sub-study will be performed with patients of both groups to compare their experiences.

\subsection{Effects and Risks}

Benefit of study participation: Patients in both groups will learn how to measure and document their pain and associated symptoms. Patients in the intervention group will obtain new knowledge about their pain and will receive self-management support. As soon as the six-week study period following discharge is over and the qualitative interviews have been completed, patients in the control group will also be offered the intervention.

Potential risks of study participation: During the qualitative interviews, patients may experience strong emotions that are related to their pain and disease. In this case, they will be offered to interrupt or stop the interview. Patients in the intervention group may take more analgesics and experience more side effects. However, pain treatment will be prescribed according to state-ofthe-art guidelines [16] and patients will be supported in monitoring and managing their side effects.

\subsection{Sample Size Calculation}

Assuming the previous study's attrition rate of $18 \%$ at six weeks, 48 adult oncology in-patients will be recruited to achieve a sample of $\mathrm{N}=40$ patients with full data sets at the end of the study. Over the last 6 months, the palliative care consultation service consulted approximately 35 patients with cancer-related pain. Assuming a response rate of $70 \%$, a recruitment period of 12 months seems reasonable.

\subsection{Methods of Randomization}

After informed consent and baseline data collection, patients will be randomized 1:1 into the intervention and the control group. Group allocation will be concealed from study personnel by using a computer generated list with randomly permuting block lengths. A study nurse will prepare opaque envelops, which will be ordered and kept in a box accessible to recruiting personnel.

\subsection{Inclusion Criteria}

Patients will be included if they:

- have cancer-related pain $>3$ (NRS $0=$ no pain to $10=$ worst pain imaginable),

- will be discharged from hospital and will self-manage their pain after discharge,

- have an expected life expectancy of $\geq 3$ months,

- understand, read and write German,

- and have access to a telephone.

\subsection{Exclusion Criteria}

Patients will be excluded if their treating physicians perceive that they have severe cognitive deficits. Attrition is expected to occur mostly because of deterioration of the patients' health status. Therefore, data that were collected before hospitalization will be included in the analysis unless patients explicitly withdraw their consent. 
Patients who are hospitalized for less than two weeks will resume the study after discharge.

\subsection{Study Procedures}

Recruitment: Patients will be screened for potential eligibility by the palliative care consultation service. For all patients, the palliative care consultation service will recommend state-of-the-art pain treatment [16] and contact an intervention nurse. Intervention nurses will be specifically trained Bachelor prepared nurses with profound experience in oncology nursing. The recommendation for pain treatment will be carried out by health care professionals on the wards. The intervention nurse will verify in- and exclusion criteria, inform patients about the project and invite them to participate.

Data collection: After written informed consent and before randomization, baseline data will be collected by the intervention nurse. At one and six weeks after discharge, data collection will be performed by a blinded study nurse via mail and telephone. If patients are hospitalized for more than two weeks during the six-week study period following discharge, they will be excluded from further study participation.

Interventions: Patients in the control group will not receive additional pain self-management support during the study. After the six-week study period of following discharge and the qualitative interview, patients in the control group will be offered pain self-management support by an intervention nurse.

Patients in the intervention group will be offered pain self-management support by the intervention nurse who will be required to follow a detailed intervention protocol. For patients in the intervention group, the intervention nurse will carry out the first session of the cancer pain self-management support intervention in the hospital. If a family caregiver is involved in the patient's pain selfmanagement, they will be invited to participate in the intervention. The intervention will consist of a face-toface session during the inpatient stay, follow-up phone calls, and face-to-face contacts according to the patient's further treatment schedule. Provision of information and skill building will be provided mainly during the first session, whereas the nurse coaching component will be provided mainly after discharge. Patient follow-up will continue until the pain situation has been stabilized according to a clinical algorithm based on pain intensity, satisfaction with pain management and adherence to analgesic medication and recommendations.

The intervention will be designed to provide structured and tailored components. Structured intervention components include an initial assessment of the patient's pain, side effects and experiences with pain management. Furthermore, all participants will be trained to monitor their pain, other symptoms and analgesic intake. Based on the patient's answers on a questionnaire, the intervention nurse will guide a discussion about the patient's attitudes and knowledge regarding pain management (academic detailing teaching approach [17]) and hand out a corresponding booklet. Tailored intervention components include information and practical advice about each patient's analgesic prescription and corresponding side effects, information about how to communicate with clinicians if pain control is inadequate and changes are needed in the analgesic prescription, as well as the use of a weekly pillbox.

At the end of each visit, the patient will have detailed, written pain and side-effect management plans that provide a list of the discussed strategies. In addition, a time for the next contact will be scheduled according to the criteria provided in the algorithm. At each follow-up contact, adherence to analgesic medication and the recommendations of the last contact will be assessed and discussed with the patient. In addition, the patient will be screened for side-effects and other relevant symptoms.

Quality control: All intervention nurses will follow a detailed intervention protocol. To ensure protocol fidelity, adherence of each intervention nurse will be monitored with audio tapes of the first visits. A second intervention nurse will listen to the tapes and give feedback to the intervention nurse in order to reach approximately $90 \%$ adherence to the intervention protocol. After adherence has been achieved, a random sample of $\sim 10 \%$ of the contacts will be audiotaped and controlled. These measures have been used successfully in the previous study.

\subsection{Statistical Design}

Variables and measurement: To address the first specific aim, the semi-structured field notes and qualitative interviews will be analyzed. The field notes will be taken at each contact, including time and content of the intervention as well as information about any experiences with the intervention procedures and content. The field notes and qualitative interviews will be analyzed based on the following feasibility criteria: acceptability, demand, implementation, practicality, adaptation, integration, expansion, and limited efficacy (see Table 2) [18].

For the second aim, the primary outcome will be pain intensity. We will also assess the following secondary outcomes: The number of patients with pain as chief symptom, side-effects, patient satisfaction with pain management and information about pain management, pain interference with function, adherence to analgesic medication and the intervention nurse's recommendations, quality of life, knowledge and attitudes towards pain management, as well as pain-related self-efficacy.

Outcomes will be assessed in light of effect sizes and 
Table 2. Key areas of focus for feasibility studies and possible outcomes [18].

\begin{tabular}{|c|c|c|}
\hline Area of focus & The feasibility study asks... & Selected outcomes \\
\hline Acceptability & $\begin{array}{l}\text { To what extent is a new idea, program, process or } \\
\text { measure judged as suitable, satisfying, or attractive } \\
\text { to program deliverers? To program recipients? }\end{array}$ & $\begin{array}{l}\text { - Satisfaction with pain management } \\
\text { - Intent to continue use } \\
\text { - }\end{array}$ \\
\hline Demand & $\begin{array}{l}\text { To what extent is a new idea, program, process, or } \\
\text { measure likely to be used (i.e., how much demand } \\
\text { is likely to exist?) }\end{array}$ & - $\quad$ Expressed interest or intention to use \\
\hline Implementation & $\begin{array}{l}\text { To what extent can a new idea, program, process, or measure } \\
\text { be successfully delivered to intended participants in some } \\
\text { defined but not fully controlled context? }\end{array}$ & $\begin{array}{l}\text { - } \quad \text { Degree of execution } \\
\text { - } \quad \text { Success or failure of execution } \\
\text { - }\end{array}$ \\
\hline Practicality & $\begin{array}{l}\text { To what extent can an idea, program, process, or } \\
\text { measure be carried out with intended participants } \\
\text { using existing means, resources, and circumstances } \\
\text { and without outside intervention? }\end{array}$ & $\begin{array}{l}\text { - Factors affecting implementation ease or difficulty } \\
\text { - } \quad \text { Positive/negative effects on target participants } \\
\text { - }\end{array}$ \\
\hline Adaptation & $\begin{array}{l}\text { To what extent does an existing idea, program, process, } \\
\text { or measure function when changes are made for a new } \\
\text { format or with a different population? }\end{array}$ & - Comparison between intervention group and standard care group \\
\hline Integration & $\begin{array}{l}\text { To what extent can a new idea, program, process, or } \\
\text { measure be integrated into an existing system? }\end{array}$ & \\
\hline Expansion & $\begin{array}{l}\text { To what extent can a previously tested program, } \\
\text { process, approach, or system be expanded to provide } \\
\text { a new program or service? }\end{array}$ & - Not within the scope of this study \\
\hline Limited efficacy & $\begin{array}{l}\text { Does the a new idea, program, process, or measure } \\
\text { show promise of being successful with the intended } \\
\text { population, even in a highly controlled setting? }\end{array}$ & $\begin{array}{ll}\text { - } & \text { Effect-size estimation } \\
\text { - } & \text { Perceived effect of patients in both groups }\end{array}$ \\
\hline
\end{tabular}

fit with the study aim. Demographic and clinical data, functional status, the patient's initial preference of study arm, as well as the dose of and adherence to the intervention will be measured as covariates.

Table 3 provides a listing of variables and instruments and times of measurement. Data for each patient will be collected at baseline (T0), and one (T1) and six weeks (T2) after discharge.

Demographic data will include age, gender, years of formal education (school and vocational training, university, etc.), profession, living condition (i.e., alone or not), and information about family support. Clinical data will include type of tumor, comorbidities, time since tumor diagnosis, and time since pain onset. Most information will be obtained from medical records. Additional data (e.g. educational status) will be obtained from the patients via questionnaire.

Eastern Cooperative Oncology Group Performance Status (ECOG-PS): Functional status will be measured with the ECOG, referring to the patient's capacity to perform a variety of activities that are normal for most people [19]. The German version will be used [20].

Depression will be measured with the PHQ-2, a 2-item screening tool [21]. Decrease of interest and dejectedness are measured each on 0 (never) to 3 (almost every day). Sensitivity and specificity to detect depressive disorders were $79 \%$ and $56 \%$ with a cutoff of $\geq 3$ and Crohnbach's $\alpha$ was 0.83 [21].
Brief Pain Inventory (BPI): The BPI is a comprehensive self-report questionnaire to assess pain management in 5 dimensions: pain intensity, analgesic intake, pain alleviation, pain location and pain interference with functioning [22]. Pain intensity, i.e. average, least and worst pain, and pain right now, are each measured on a 0 (none) to 10 (worst imaginable pain) NRS. Around-theclock and as-needed medication taken in the last 48 hours will be listed by the patient. Patients will rate the alleviation that they obtained from this medication on a 0 (no alleviation) to 100 (complete alleviation) scale. Patients will be asked to draw the location of their pain by shading the affected areas of their body on a body map. In addition, they will be asked to make a cross on the most affected body part. Pain interference with functioning is a 7-item scale in which patients are asked to evaluate how pain has interfered with a variety of activities during the past week using a scale from 0 (does not interfere) to 10 (completely interferes). Scores on the individual items will be summed and divided by 7 to obtain a total interference score that ranges from 0 to 10 . The BPI has established validity and reliability [23]. The $\alpha$ reliability for the scale ranges from 0.91 to 0.95 [22].

Main symptom and side-effects of pain and cancer treatment: In the Memorial Symptom Assessment Scale (MSAS), patients report the presence of 32 symptoms. The checklist has been developed for cancer patients by Portenoy et al. [24] Symptom occurrence (i.e. frequency 
Table 3. Listing of study variables and instruments.

\begin{tabular}{|c|c|c|}
\hline Variables & Measurement & $\begin{array}{c}\text { Number of items } \\
\text { filled out by patients }\end{array}$ \\
\hline Demographic data ${ }^{\mathrm{T} 0}$ & Patient information questionnaire & 10 items \\
\hline Clinical data ${ }^{\mathrm{T}}$ & Medical record review form & \\
\hline Functional status ${ }^{\mathrm{T} 0, \mathrm{~T} 1, \mathrm{~T} 2}$ & Eastern Cooperative Oncology Group Performance Status & 1 item \\
\hline Depression $^{\mathrm{T} 0, \mathrm{~T} 1, \mathrm{~T} 2}$ & PHQ-2 & 2 items \\
\hline $\begin{array}{l}\text { Average and worst pain intensity }{ }^{\mathrm{T} 0, \mathrm{~T} 1, \mathrm{~T} 2} \\
\text { Around-the-clock and "as-needed" analgesic medication } \\
\text { Pain alleviation from pain medication } \\
\text { Pain location } \\
\text { Pain interference with functioning }\end{array}$ & Brief Pain Inventory & 12 items \\
\hline Side-effects of pain and cancer treatment ${ }^{\mathrm{T} 0, \mathrm{~T} 1, \mathrm{~T} 2}$ & Memorial Symptom Assessment Scale (MSAS) & 35 items \\
\hline Main symptoms ${ }^{\mathrm{T} 0, \mathrm{~T} 1, \mathrm{~T} 2}$ & Rank order question of 3 main symptoms of the MSAS & 3 items \\
\hline Patient satisfaction with pain management ${ }^{\mathrm{T} 0, \mathrm{~T} 1, \mathrm{~T} 2}$ & 0 - 10 numeric rating scale; self-developed & 1 item \\
\hline Patient satisfaction with information about pain treatment ${ }^{\mathrm{T} 1}$ & 0 - 10 numeric rating scale; self-developed & 1 item \\
\hline Adherence to analgesic therapy ${ }^{\mathrm{T} 1, \mathrm{~T} 2}$ & 0 - 100 numeric rating scale & 1 item \\
\hline Adherence to recommendations of intervention nurse ${ }^{\mathrm{T} 1, \mathrm{~T} 2}$ & 0 - 100 numeric rating scale & 1 item \\
\hline Quality of life ${ }^{\mathrm{T} 0, \mathrm{~T} 1, \mathrm{~T} 2}$ & Medical Outcomes Study-Short Form (SF-12) & 12 items \\
\hline Knowledge of cancer pain ${ }^{\mathrm{T} 0, \mathrm{~T} 1, \mathrm{~T} 2}$ & Barriers Questionnaire (BQ II) & 27 items \\
\hline Importance of analgesic intake ${ }^{\mathrm{T} 0, \mathrm{~T} 1, \mathrm{~T} 2}$ & 0 - 10 numeric rating scale; self-developed* & 1 item \\
\hline Pain-related self-efficacy & 0 - 6 numeric rating scale (FESS) & 10 items \\
\hline The pain hindered me to do things that I wanted to do today ${ }^{\mathrm{T} 1, \mathrm{~T} 2}$ & 0 to 10 numeric rating scale; self-developed & 1 item \\
\hline Patient preference for study arm ${ }^{\mathrm{T} 0}$ & $10 \mathrm{~cm}$ visual analogue scale; self-developed & 1 item \\
\hline \multicolumn{3}{|l|}{ Variables derived from analgesic intake } \\
\hline Adequacy of analgesic prescription ${ }^{\mathrm{T} 0, \mathrm{~T} 1, \mathrm{~T} 2}$ & Pain Management Index (PMI) & \\
\hline Opioid intake $\mathrm{T}^{\mathrm{T}, \mathrm{T} 1, \mathrm{~T} 2}$ & Morphine equivalent & \\
\hline Complexity of analgesic regimen & $\begin{array}{l}\text { Number of different analgesics } \\
\text { Number of time-points of analgesic intake } \\
\text { Number of other medications }\end{array}$ & \\
\hline \multicolumn{3}{|c|}{ Additional variables for intervention and post intervention data collection ${ }^{\mathrm{I}}$} \\
\hline Time spent with intervention nurse & In minutes & \\
\hline Dose of intervention: topics covered & Checklist of topics covered during intervention & \\
\hline Dose of intervention: duration & Time spent & \\
\hline Adherence to the intervention & Checklist of pain management strategies; yes, no & \\
\hline
\end{tabular}

${ }^{\mathrm{T} 0}$ collected at baseline; ${ }^{\mathrm{T} 1}$ collected at T1, one week after discharge; ${ }^{\mathrm{T} 2}$ collected at T3, six weeks after discharge; ${ }^{\mathrm{I}}$ collected at interventions; *rated independently by the patient and the study nurse

and severity) and symptom distress are rated for present symptoms. Eight symptoms are evaluated only in terms of severity and distress since using a frequency scale is not meaningful (e.g. weight loss). Three additional lines allow for patients to list symptoms that are not included. Symptoms are scored on a four-point Likert scale (1 - 4) for frequency and severity, and on a five-point Likert scale $(0$ - 4) for distress. In this study, the German ver- sion of Spichiger et al. [20] will be used. Patients will be asked if they have one, two, three or no main symptoms. They will then be asked to rank these by putting the numbers 1 (main symptom) to 3 (third main symptom) next to the symptoms.

Patient satisfaction with pain management will be assessed with an 11-point NRS (0 = not satisfied at all to $10=$ completely satisfied) at T1. Patient satisfaction with 
information about pain treatment will be assessed with an 11-point NRS ( $0=$ not satisfied at all to $10=$ completely satisfied) at $\mathrm{T} 2$ and $\mathrm{T} 3$.

Adherence to prescribed analgesics and the intervention: Patient self-report is a useful method to assess adherence to self-management support interventions [25]. Patients will rate their adherence to the medical prescription and adherence to the noted recommendations for pain self-management from the previous visit with a NRS respectively $(0=$ no adherence at all; $100=$ complete adherence).

Medical Outcomes Study-Short Form (SF-12): This 12-item instrument is designed to measure quality of life by assessing health concepts that are relevant to a person's functional status and well-being. The SF-12, including the German version, has been demonstrated to be reliable and valid in clinical and population-based applications in the U.S. and other countries [26-29].

Knowledge of and attitudes towards cancer pain: These variables will be evaluated with the Barriers Questionnaire-II (BQ-II), which is a self-report instrument originally developed in 1993[30]. After its revision [31], the BQ-II, consisted of 27 items addressing eight common barriers (i.e., concerns about addiction, tolerance, side effects, about distracting the physician from treating the cancer, that pain medications may impair the immune system, and analgesics may mask pain as sign of warning; fatalism; and the belief that "good" patients do not complain about pain). Items are scored on 6-point Likert scales anchored with 0 (do not agree at all), and 5 (agree very much). The BQ-II showed a high level of internal consistency ( $\alpha=0.9$ ) [31]. In addition, the importance of taking their analgesic medication will be assessed in patients with acute or imminent pain by asking, "How important do you think is it for you at the moment to take your analgesic medication?” on a scale of 0 (not at all) to 10 (extremely important). Based on the patients' pain levels and the potential reasons for having pain, the study nurse will independently rate her view of the importance of that patient taking their analgesic medication on a scale of 0 (not important) to 10 (extremely important) . The nurse will also write down a short clinical reasoning for her judgment.

Self-efficacy will be measured with the German adaptation of the Pain Self-efficacy Questionnaire (FESS) [32]. The FESS is a 10-item scale. Patients rate each of the items regarding their perceived ability to manage various aspects of their pain on scales of 0 (very uncertain) to 6 (very certain). Crohnbach's alpha for the German version was 0.93 [32]. In addition, we use the self-developed item from our previous study [12], by asking the patients to rate "The pain hindered me to do things that I wanted to do today." on a 0 (not at all) to 10 (completely) scale.
Preference for study arm: To assess whether patient preference for the intervention has an influence on outcomes, the patients' degree of preference for the intervention or the wait-list control study arm will be assessed using a $10 \mathrm{~cm}$ long visual analogue scale (left end: "I would absolutely prefer to be in the intervention group”, right end "I would absolutely prefer to be in the wait-list control group" and an indication at $5 \mathrm{~cm}$ marked as "I do not have a preference for either of the two groups"). Before answering the question, patients will be informed that their preference will not have an effect on their randomization in this pilot study but that the results of this item will be used to plan the design of a larger study [33].

Variables derived from analgesic intake: The Pain Management Index (PMI) is a composite measure to assess the adequacy of analgesic prescription. It is computed by subtracting a patient's worst pain intensity from the rating of the most potent analgesic prescribed [34]. Scores can range from -3 to 3 , with negative scores indicating low adequacy. The PMI is one of the most used outcome measures for quality of pain treatment [35-37].

Opioid intake per day: All doses of opioids will be converted to morphine equivalents per 24 hours. Total and ATC doses of the opioid analgesics taken by patients will be calculated.

Complexity of analgesic regimen: The number of different analgesics, number of points in time as well as the number of other medications will be calculated for each patient.

Data analysis: Data files will be analyzed using IBM SPSS Statistics 19.0. Data will be systematically examined for out of range values and data inconsistencies. Appropriate descriptive statistics will be calculated for all study variables. The analysis will follow an intent-to-treat strategy with a level of significance at 0.05 . Although withdrawal is expected to be a random process, participants who completed the study and those who did not will be compared based on demographics, treatment group and other salient variables. Change scores for each patient as well as Cohen's d will be calculated for each variable of interest. To determine differences between the change scores of the pre- and the post-assessments regarding average and worst pain intensity between the two groups, regression analysis will be applied.

\subsection{Qualitative Substudy}

Recruitment: A sample of 10 - 12 patients seems reasonable to reach sufficient redundancy in the data. In the beginning, consecutive patients will be invited to participate. Later, purposive sampling will be used to ensure variation (e.g., regarding age, gender, education, occupation, living situation, tumor entity and disease status). 
Table 4. Main questions for semi-structured interview guide (Patients of both groups will be asked the same questions).

\begin{tabular}{ll}
\hline $\begin{array}{l}\text { Opening generic question } \\
\text { Follow-up questions }\end{array}$ & $\begin{array}{l}\text { Maybe we could start with how you experienced your pain management during the study?* } \\
\text { How was the pain management shortly before discharge? How was it after you were discharged? } \\
\text { In your opinion, has the study influenced the way you dealt with your pain at home? If it did, in which way? }\end{array}$ \\
$\begin{array}{ll}\text { Intervention procedures } \\
\text { Follow-up questions }\end{array}$ & $\begin{array}{l}\text { When you think of your first visit with Mrs. XX before you were discharged, what was your experience then? } \\
\text { After you were discharged, how did the study go on for you? }\end{array}$ \\
\hline & $\begin{array}{l}\text { Were there any organizational issues while taking part in the study? } \\
\text { What did you think of the burden and benefit of study participation? }\end{array}$ \\
Study procedures & $\begin{array}{l}\text { For the study, you filled out several questionnaires, what did you think of that? } \\
\text { Follow-up questions } \\
\text { There there any questions difficult to answer in the questionnaires? } \\
\text { "The pain hindered me to do the things that I wanted to do today" }\end{array}$ \\
\hline Closing remarks & $\begin{array}{l}\text { Do you have anything else that you would like to tell the study leader? } \\
\text { If you had a close friend with cancer-related pain, would you recommend taking part in the study? }\end{array}$
\end{tabular}

*The leading question is printed bold; follow-up questions will only be asked if these topics have not been covered spontaneously by the participants. The general interview technique will include questions like "Can you think of anything else?" or "Could you tell me some more details about this?" to maintain the participants' flow of speech. Each leading question will be printed on a card together with the follow-up questions to guide the interview nurse during the interview.

Data collection: Patients of both groups will be interviewed according to a semi-structured interview guide by a study nurse who has not been involved in the intervention. The interview will start with a generic question about the participants' experience with their pain management during the study period (see Table 4). More focused questions will be asked about their experiences with the feasibility of the intervention and their view of the data collection procedures. Interview nurses will be trained on how to interview patients according to the interview guide and how to maintain a flow of speech.

Data analysis: The interviews will be audio recorded, transcribed verbatim and analyzed using qualitative content analysis as described by Mayring [38]. This will include a stepwise and systematic processing and reduction of data leading to a meaningful synopsis. After the first interview, the project leader will listen to the tapes and give feedback to the interview nurse. Ongoing transcription and analysis during the recruitment period will allow adaptation of the interview guide during the study if necessary.

\subsection{Ethical and Legal Aspects}

- The study will be performed according to the Declaration of Helsinki.

- Ethical approval has been obtained from the local ethical board.

- Participation of patients is entirely voluntary. Patients are free to withdraw their consent at any time without any disadvantages for their medical treatment or nursing care. Patients will give written, informed consent after comprehensive oral and written information. Furthermore, patients will be informed that already collected data will be used in pseudomized form if they do not explicitly state otherwise.

- Data will be pseudomized by using a numerical ID. The master file with patient names and ID number will be kept on local servers of the clinic and will be locked via a password that will only be known by the project leader and her substitutes.

\section{CONCLUSION}

The results of this pilot study will build the basis for a larger comparative effectiveness study in which long term outcomes of a cancer pain self-management intervention will be evaluated with a strong focus on feasibility in clinical practice.

\section{ACKNOWLEDGEMENTS}

This research is funded through the program "Development and coordination of health services research in Baden-Württemberg” [Ausbau und Koordination der Versorgungsforschung in Baden-Württemberg], especially the "Junior researcher academy related to health services research” [Nachwuchsakademie Versorgungsforschung]. The article processing charge was funded by the German Research Foundation (DFG) and the Albert Ludwigs University Freiburg through the funding program Open Access Publishing. Furthermore we greatly thank Professor Christine Miaskowski for collaborating with us on the refinement of the PRO-Self ${ }^{\odot}$ Plus PCP for the German clinical context.

Biometrician: Prof. Werner Vach; Clinical Epidemiology and Coordinating Office for Health Service Research, Department of Medical Biometry and Medical Informatics, University Medical Center Freiburg, Stefan Meier Str. 26, 79104 Freiburg.

\section{REFERENCES}

[1] Van den Beuken-van Everdingen, M.H., de Rijke, J.M., Kessels, A.G., Schouten, H.C., van Kleef, M. and Patijn, J. (2007) Prevalence of pain in patients with cancer: A systematic review of the past 40 years. Annals of Oncology, 18, 1437-1449.

[2] Mercadante, S. (2007) Why are our patients still suffering pain? Nature Clinical Practice Oncology, 4, 138-139. http://dx.doi.org/10.1038/ncponc0745 
[3] Breivik, H., Cherny, N., Collett, B., de Conno, F., Filbet, M., Foubert, A.J., Cohen, R. and Dow, L. (2009) Cancerrelated pain: A pan-European survey of prevalence, treatment, and patient attitudes. Annals of Oncology, 20, 14201433. http://dx.doi.org/10.1093/annonc/mdp001

[4] Jacobsen, R., Liubarskiene, Z., Moldrup, C., Christrup, L., Sjogren, P. and Samsanaviciene, J. (2009) Barriers to cancer pain management: A review of empirical research. Medicina (Kaunas), 45, 427-433.

[5] Allard, P., Maunsell, E., Labbe, J. and Dorval, M. (2001) Educational interventions to improve cancer pain control: A systematic review. Journal of Palliative Medicine, 4, 191-203. http://dx.doi.org/10.1089/109662101750290227

[6] Bennett, M.I., Bagnall, A.M. and Closs, S.J. (2009) How effective are patient-based educational interventions in the management of cancer pain? Systematic review and meta-analysis. Pain, 143, 192-199.

http://dx.doi.org/10.1016/j.pain.2009.01.016

[7] Devine, E.C. (2003) Meta-analysis of the effect of psychoeducational interventions on pain in adults with cancer. Oncology Nursing Forum, 30, 75-89. http://dx.doi.org/10.1188/03.ONF.75-89

[8] Koller, A., Miaskowski, C., De Geest, S., Opitz, O. and Spichiger, E. (2013) A systematic evaluation of content, structure, and efficacy of interventions to improve patients' self-management of cancer pain. Journal of Pain and Symptom Management, 44, 264-284. http://dx.doi.org/10.1016/j.jpainsymman.2011.08.015

[9] Miaskowski, C., Dodd, M., West, C., Schumacher, K., Paul, S.M., Tripathy, D. and Koo, P. (2004) Randomized clinical trial of the effectiveness of a self-care intervention to improve cancer pain management. Journal of Clinical Oncology, 22, 1713-1720.

http://dx.doi.org/10.1200/JCO.2004.06.140

[10] Miaskowski, C., Dodd, M., West, C., Paul, S.M., Schumacher, K., Tripathy, D. and Koo, P. (2007) The use of a responder analysis to identify differences in patient outcomes following a self-care intervention to improve cancer pain management. Pain, 129, 55-63. http://dx.doi.org/10.1016/j.pain.2006.09.031

[11] Schumacher, K.L., Koresawa, S., West, C., Hawkins, C., Johnson, C., Wais, E., Dodd, M., Paul, S.M., Tripathy, D., Koo, P. and Miaskowski, C. (2002) Putting cancer pain management regimens into practice at home. Journal of Pain and Symptom Management, 23, 369-382. http://dx.doi.org/10.1016/S0885-3924(02)00385-8

[12] Koller, A., Miaskowski, C., De Geest, S., Opitz, O. and Spichiger, E. (2013) Results of a randomized controlled pilot study of a self-management intervention for cancer pain. European Journal of Oncology Nursing, 17, 284291. http://dx.doi.org/10.1016/j.ejon.2012.08.002

[13] Koller, A., Miaskowski, C., De Geest, S., Opitz, O. and Spichiger, E. (2013) Supporting self-management of pain in cancer patients: Methods and lessons learned from a randomized controlled pilot study. European Journal of Oncology Nursing, 17, 1-8.

http://dx.doi.org/10.1016/j.ejon.2012.02.006

[14] Koller, A., Miaskowski, C., De Geest, S., Opitz, O. and Spichiger, E. (2012) Testing of an intervention to support pain self-management in oncology patients: A mixed methods pilot study. Pflege: Die wissenschaftliche Zeitschrift für Pflegeberufe, 25, 305-306.

[15] Luescher, C., Koller, A., Schaefer, I. and Spichiger, E. (2012) Oncological patients' decision making processes concerning their pain medication at home: A qualitative secondary analysis. Pflege: Die wissenschaftliche Zeitschrift für Pflegeberufe, 25, 353-362.

[16] National Guideline Clearinghouse (2010) Cancer pain management (general). In: Guidelines on Pain Management, Agency for Healthcare Research and Quality (AHRQ), Rockville.

[17] Soumerai, S.B. and Avorn, J. (1990) Principles of educational outreach ("academic detailing") to improve clinical decision making. Journal of the American Medical Association, 263, 549-556. http://dx.doi.org/10.1001/jama.1990.03440040088034

[18] Bowen, D.J., Kreuter, M., Spring, B., Cofta-Woerpel, L., Linnan, L., Weiner, D., Bakken, S., Kaplan, C.P., Squiers, L., Fabrizio, C. and Fernandez, M. (2009) How we design feasibility studies. American Journal of Preventive Medicine, 36, 452-457. http://dx.doi.org/10.1016/j.amepre.2009.02.002

[19] Oken, M.M., Creech, R.H., Tormey, D.C., Horton, J., Davis, T.E., McFadden, E.T. and Carbone, P.P. (1982) Toxicity and response criteria of the Eastern Cooperative Oncology Group. American Journal of Clinical Oncology, 5, 649-655. http://dx.doi.org/10.1097/00000421-198212000-00014

[20] Spichiger, E., Muller-Frohlich, C., Denhaerynck, K., Stoll, H., Hantikainen, V. and Dodd, M. (2011) Symptom prevalence and changes of symptoms over ten days in hospitalized patients with advanced cancer: A descriptive study. European Journal of Oncology Nursing, 15, 95102. http://dx.doi.org/10.1016/j.ejon.2010.06.005

[21] Lowe, B., Kroenke, K. and Grafe, K. (2005) Detecting and monitoring depression with a two-item questionnaire (PHQ-2). Journal of Psychosomatic Research, 58, 163171. http://dx.doi.org/10.1016/j.jpsychores.2004.09.006

[22] Radbruch, L., Loick, G., Kiencke, P., Lindena, G., Sabatowski, R., Grond, S., Lehmann, K.A. and Cleeland, C.S. (1999) Validation of the German version of the brief pain inventory. Journal of Pain and Symptom Management, 18, 180-187. http://dx.doi.org/10.1016/S0885-3924(99)00064-0

[23] Jensen, M.P. (2003) The validity and reliability of pain measures in adults with cancer. Journal of Pain, 4, 2-21. http://dx.doi.org/10.1054/jpai.2003.1

[24] Portenoy, R.K., Thaler, H.T., Kornblith, A.B., Lepore, J.M., Friedlander-Klar, H., Kiyasu, E., Sobel, K., Coyle, N., Kemeny, N., Norton, L., et al. (1994) The memorial symptom assessment scale: An instrument for the evaluation of symptom prevalence, characteristics and distress. European Journal of Cancer, 30A, 1326-1336. http://dx.doi.org/10.1016/0959-8049(94)90182-1

[25] Vitolins, M.Z., Rand, C.S., Rapp, S.R., Ribisl, P.M. and Sevick, M.A. (2000) Measuring adherence to behavioral and medical interventions, Controlled Clinical Trials, 21, 188S-194S. 
http://dx.doi.org/10.1016/S0197-2456(00)00077-5

[26] Gandek, B., Ware, J.E., Aaronson, N.K., Apolone, G., Bjorner, J.B., Brazier, J.E., Bullinger, M., Kaasa, S., Leplege, A., Prieto, L. and Sullivan, M. (1998) Cross-validation of item selection and scoring for the SF-12 Health Survey in nine countries: Results from the IQOLA Project. International Quality of Life Assessment. Journal of Clinical Epidemiology, 51, 1171-1178. http://dx.doi.org/10.1016/S0895-4356(98)00109-7

[27] Lundberg, L., Johannesson, M., Isacson, D.G. and Borgquist, L. (1999) The relationship between health-state utilities and the SF-12 in a general population. Medical Decision Making, 19, 128-140. http://dx.doi.org/10.1177/0272989X9901900203

[28] Ware Jr., J., Kosinski, M. and Keller, S.D. (1996) A 12item short-form health survey: Construction of scales and preliminary tests of reliability and validity. Medical Care, 34, 220-233. http://dx.doi.org/10.1097/00005650-199603000-00003

[29] Bullinger, M. (1995) German translation and psychometric testing of the SF-36 health survey: Preliminary results from the IQOLA Project. International Quality of Life Assessment, Social Science and Medicine, 41, 13591366. http://dx.doi.org/10.1016/0277-9536(95)00115-N

[30] Ward, S.E., Goldberg, N., Miller-McCauley, V., Mueller, C., Nolan, A., Pawlik-Plank, D., Robbins, A., Stormoen, D. and Weissman, D.E. (1993) Patient-related barriers to management of cancer pain. Pain, 52, 319-324. http://dx.doi.org/10.1016/0304-3959(93)90165-L

[31] Gunnarsdottir, S., Donovan, H.S., Serlin, R.C., Voge, C. and Ward, S. (2002) Patient-related barriers to pain man-

\section{LIST OF ABBREVIATIONS}

ATC: around the clock

BPI: Brief Pain Inventory

BQ-II: Barriers Questionnaire-II

CCCF: Tumorzentrum Ludwig Heilmeyer - Comprehensive Cancer Center Freiburg

ECOG-PS: Eastern Cooperative Oncology Group Performance Status

FC: family caregiver

FESS: Pain Self-efficacy Questionnaire

MSAS: Memorial Symptom Assessment Scale agement: The Barriers Questionnaire II (BQ-II). Pain, 99, 385-396.

http://dx.doi.org/10.1016/S0304-3959(02)00243-9

[32] Mangels, M., Schwarz, S., Sohr, G., Holme, M. and Rief, W. (2009) An adaptation of the pain self-efficacy quesionnaire for the German speaking context. Diagnostica, 55, 84-93. http://dx.doi.org/10.1026/0012-1924.55.2.84

[33] Torgerson, D.J. and Sibbald, B. (1998) Understanding controlled trials. What is a patient preference trial? British Medical Journal, 316, 360.

http://dx.doi.org/10.1136/bmj.316.7128.360

[34] Cleeland, C.S., Gonin, R., Hatfield, A.K., Edmonson, J.H., Blum, R.H., Stewart, J.A. and Pandya, K.J. (1994) Pain and its treatment in outpatients with metastatic cancer. New England Journal of Medicine, 330, 592-596. http://dx.doi.org/10.1056/NEJM199403033300902

[35] Beck, S.L. and Falkson, G. (2001) Prevalence and management of cancer pain in South Africa. Pain, 94, 75-84. http://dx.doi.org/10.1016/S0304-3959(01)00343-8

[36] Hyun, M.S., Lee, J.L., Lee, K.H., Shin, S.O., Kwon, K.Y., Song, H.S., Kim, O.B., Sohn, S.K., Lee, K.B., Rhu, H.M., Park, G.W. and Shin, D.G. (2003) Pain and its treatment in patients with cancer in Korea. Oncology, 64, 237-244. http://dx.doi.org/10.1159/000069314

[37] Sabatowski, R., Arens, E.R., Waap, I. and Radbruch, L. (2001) Cancer pain management in Germany-results and analysis of a questionnaire. Schmerz, 15, 241-247. http://dx.doi.org/10.1007/s004820100054

[38] Mayring, P. (2010) Qualitative content analysis: Basic principles and techniques. 11th Edition, Beltz, Weinheim.

NRS: numeric rating scale

PHQ 2: Patient Health Questionnaire Depression short form

PI: Pain Management Index

PRO-Self@ Plus PCP: PRO-Self@ Plus Pain Control Program

RCT: randomized controlled trial

SF-12: Medical Outcomes Study-Short Form

T0: baseline

T1: one week after discharge

T2: six weeks after discharge 